\title{
Automated Quantification of Retinal Microvasculature from OCT Angiography using Dictionary-Based Vessel Segmentation
}

Engberg, Astrid Margareta Elisabet; Erichsen, Jesper H.; Sander, Birgit; Kessel, Line; Dahl, Anders Bjorholm; Dahl, Vedrana Andersen

Published in:

Medical Image Understanding and Analysis

Link to article, DOI:

10.1007/978-3-030-39343-4_22

Publication date:

2019

Document Version

Peer reviewed version

Link back to DTU Orbit

Citation (APA):

Engberg, A. M. E., Erichsen, J. H., Sander, B., Kessel, L., Dahl, A. B., \& Dahl, V. A. (2019). Automated Quantification of Retinal Microvasculature from OCT Angiography using Dictionary-Based Vessel Segmentation. In Medical Image Understanding and Analysis (pp. 257-269). Springer. Communications in Computer and Information Science Vol. 1065 https://doi.org/10.1007/978-3-030-39343-4_22

\section{General rights}

Copyright and moral rights for the publications made accessible in the public portal are retained by the authors and/or other copyright owners and it is a condition of accessing publications that users recognise and abide by the legal requirements associated with these rights.

- Users may download and print one copy of any publication from the public portal for the purpose of private study or research.

- You may not further distribute the material or use it for any profit-making activity or commercial gain

- You may freely distribute the URL identifying the publication in the public portal 


\title{
Automated Quantification of Retinal Microvasculature from OCT Angiography using Dictionary-Based Vessel Segmentation
}

\author{
Astrid M. E. Engberg' ${ }^{1}$, Jesper H. Erichsen ${ }^{2}$, Birgit Sander ${ }^{2}$, Line Kessel ${ }^{2,3}$, \\ Anders B. Dahl ${ }^{1}$, and Vedrana A. Dahl ${ }^{1}$ \\ 1 Technical University of Denmark, Kgs. Lyngby, Denmark \\ \{asteng, abda, vand\}@dtu.dk \\ 2 The Eye Clinic, Rigshospitalet-Glostrup, Denmark \\ 3 Faculty of Health and Medical Sciences, University of Copenhagen, Denmark
}

\begin{abstract}
Investigations in how the retinal microvasculature correlates with ophthalmological conditions necessitate a method for measuring the microvasculature. Optical coherence tomography angiography (OCTA) depicts the superficial and the deep layer of the retina, but quantification of the microvascular network is still needed. Here, we propose an automatic quantitative analysis of the retinal microvasculature. We use a dictionary-based segmentation to detect larger vessels and capillaries in the retina and we extract features such as densities and vessel radius. The method is validated on repeated OCTA scans from healthy subjects, and we observe high intraclass correlation coefficients and high agreement in a Bland-Altman analysis. The quantification method is also applied to pre- and postoperative scans of cataract patients. Here, we observe a higher variation between the measurements, which can be explained by the greater variation in scan quality. Statistical tests of both the healthy subjects and cataract patients show that our method is able to differentiate subjects based on the extracted microvascular features.
\end{abstract}

Keywords: OCTA · Dictionary-based segmentation · Quantification.

\section{Introduction}

An important function of the retinal microvasculature is to supply the inner retinal layers with nutrients and oxygen. Disturbances in the vasculature occur in many eye diseases. Hence, the state of the retinal microvasculature could be an important health indicator for certain eye-related conditions. One of the specific clinical problems motivating our work is assessing the risk for developing pseudophakic macular edema after cataract surgery. Our future objective is to investigate the influence of parameters related to the risk of edema development. We are therefore in need of a method to characterize the retinal microvasculature.

The retinal microvasculature is visible in images captured using the noninvasive modality, Optical Coherence Tomography Angiography (OCTA). The visualisation of the microvasculature is often susceptible to noise, potentially 
leading to misinterpretation by the operator. For the same reason, automated, clinically useful methods for extracting the vasculature from OCTA images are scarce. Methods based on user-provided annotations of various objects in large image data sets are often capable of handling a significant noise level. Unfortunately, obtaining sufficient pre-annotated data is time consuming and therefore costly.

In this paper, we present an approach for quantifying retinal microvasculature from OCTA images which utilizes a very limited amount of pre-annotated data. We present an investigation of the method with a focus on reproducibility and feasibility of extracting microvascular features.

\section{$1.1 \quad$ Related work}

Since the first clinical studies on OCTA were published in 2014, several quantification schemes for OCTA images have been invented with varying complexity and quality. Most studies use their own quantification algorithm, where the vessel detections vary from using global thresholding [11], binarization and skeletonization [12], to more complex filtering and thresholding approaches [3, 14]. More advanced methods include local fractal dimension [9], and a probabilistic model utilizing the 3D spatial information from both the superficial and the deep layer [8]. Some OCTA devices provide proprietary software for extracting vessel densities, e.g. the AngioVue OCTA system (Optovue, Inc., Fremont, CA, USA) [16], Cirrus (Carl Zeiss Meditec, Inc., Dublin, CA, USA) [7], and RS-3000 Advance (NIDEK, Gamagori, Japan) [1].

Two groups compare available macular vessel density algorithms $[18,20]$. Shoji et al. [20] assess binarization algorithms in ImageJ (National Institutes of Health, Bethesda, MD, USA) on data obtained from two OCTA systems, while Rabiolo et al. [18] compare seven previously published quantification algorithms, concluding that the estimated densities are significantly different for each algorithm. Both studies therefore recommend using the same quantification algorithm in longitudinal follow-up, and note that vessel density is dependent on both the OCTA device, the acquisition size, and the post-processing algorithm, and hence comparisons to other studies or databases should be done with care.

Due to the lack of a standardized quantification method, we choose to develop an algorithm that in later work can be used for assessing the risk of edema development. In our detection we differ between capillaries and larger vessels. This is because larger vessels influence the estimate of the capillary density. Other groups try to overcome this by filtering [2] or computing the skeleton density $[14,18]$. Our approach allows for more precise description of the vasculature.

\section{Proposed approach}

We propose using a dictionary-based method for segmenting and detecting the vascular network in the parafoveal region, i.e. the region around fovea. Our approach is motivated by the assumption that the appearance of the vascular 
network may be described as a combination of a limited number of characteristic image features.

\subsection{Dictionary-based segmentation}

The success of the quantification highly relies on its ability to detect capillaries and larger vessels. We are using a probabilistic pixel classification method for segmenting the OCTA images $[5,6]$, where pre-segmented data is used to learn a dictionary of image patches with corresponding label information. The method was originally developed for texture segmentation [4].

In short, the dictionary-based method involves two steps: building the dictionary by using pre-annotated data, and processing previously unseen images. Building the dictionary relies on two processes: defining dictionary clusters which encode local appearance of OCTA images, and learning the desired segmentation for the dictionary clusters by incorporating manual annotations. In our case, the pre-annotated data consists of one training image. For the clustering process, we extract local features from the training image. We choose to use the following features: the intensities from a patch around a pixel, and the first and the second degree image derivatives computed in a patch around a pixel. To reduce the dimensionality of the feature vectors, we perform principal component analysis (PCA) on the set of features from the training image. Once we have extracted the local features, we perform $k$-means clustering to obtain cluster centers, which in our context constitute the dictionary. The assumption behind clustering is that the variability in the local appearance of retinal images may be explained using a limited set of clusters. The learning process is performed by extending the dictionary with user-provided labeling. For each cluster in the dictionary, we access the pixels belonging to this cluster, and average the available pre-annotated information. The assumption behind learning is that pixels belonging to the same cluster should have a similar segmentation.

The segmentation of the unseen image is now performed by extracting local features and assigning them to the dictionary clusters. Then we obtain the desired segmentation information from the dictionary. We refer the reader to [6] for details.

Choosing the appropriate parameters In our dictionary for retinal microvasculature, we work with three classes: capillaries, larger vessels (arterioles and venules), and background. Larger vessels are defined as vessels of twice (or more) the radius of the capillaries. Similarly to the study by Eladawi et al. [8], manual annotations are produced and used as ground truth (GT). Two images acquired as described in section 3.1 were annotated manually by AMEE. One image is used for creating the dictionary (Fig. 1a-b), and the other is used for choosing the parameters and for validation (Fig. 1c-e).

The dictionary is built using the features from both the image seen in Fig. 1a and from a 90 degree rotated version. We use 50.000 patches of size $7 \times 7$ pixels to build the PCA co-variance matrix. The 10 biggest PCA features are 


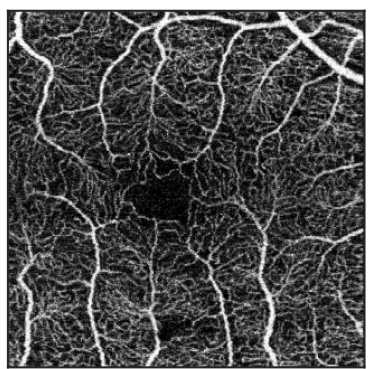

(a) Training image

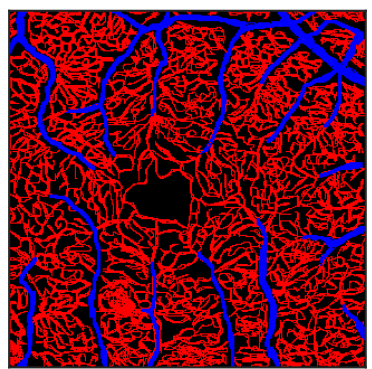

(b) GT

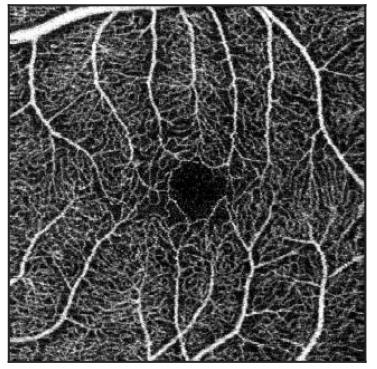

(c) Validation image

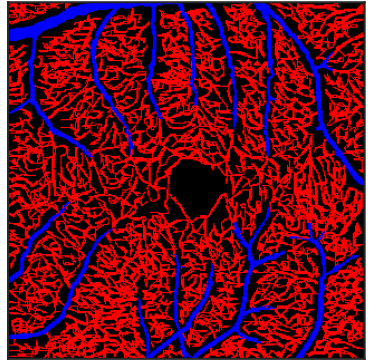

(d) GT

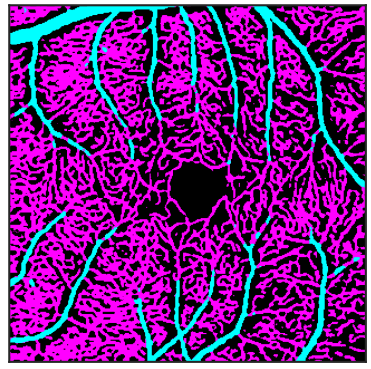

(e) Segmentation output

Fig. 1. Subfigures (a) and (b) show the image used for building the dictionary and corresponding ground truth annotation. Subfigures (c) and (d) show the validation image and corresponding manual annotation used for choosing model parameters. Subfigure (e) shows the segmentation output of the validation image.

kept. Afterwards, we build a $k$-means tree with 5 layers and a branching factor of 5 from 100.000 training patches of size $13 \times 13$ pixels. Further, we compute a $3 \times 3$ weight matrix such that the resulting class probabilities of the training image equal the annotated class labels.

We optimize the parameters for the PCA patch size and the cluster patch size with respect to the validation image and GT image shown on Fig. 1c-d. Dice scores between the manual segmentation (Fig. 1d) and the resulting segmentation (Fig. 1e) are 0.82 for larger vessels, 0.71 for capillaries, and 0.76 for background. The corresponding Jaccard scores are 0.70, 0.56, and 0.62 .

\subsection{Quantitative analysis (values VD, CD, BD, and VR)}

We extract different measures in order to quantify the vascular network in the superficial retinal layer (SRL) and the deep retinal layer (DRL) of OCTA data. We divide the parafoveal area into sections using the Early Treatment Diabetic Retinopathy Study (ETDRS) grid [10], see Fig. 2a. Due to the size of the OCTA images, the area of interest was limited to the circular radius of $1 \mathrm{~mm}$ from the foveal center. In each section, we analyze the densities of capillaries, larger vessels and background, and compute the mean radius of the larger vessels. 


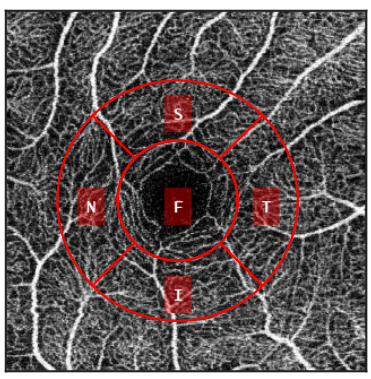

(a) ETDRS grid

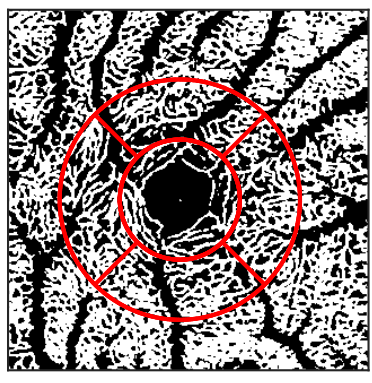

(b) Segmented capillaries

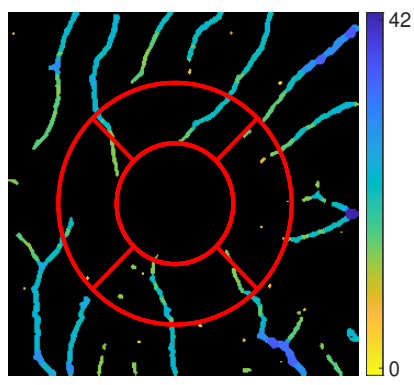

(c) Vessel radius in $\mu \mathrm{m}$.

Fig. 2. Illustration of the quantification. Subfigure (a) shows the modified ETDRS grid where the foveal center is defined as the center of the avascular zone, the radius of the central area is $0.5 \mathrm{~mm}$, and the parafoveal zone is defined as the annulus with radius $0.5 \mathrm{~mm}$ and $1 \mathrm{~mm}$. Letters F, S, I, N and T stand for fovea, superior, inferior, nasal and temporal, respectively. Subfigure (b) shows detected capillaries used to compute the capillary density. Subfigure (c) shows the radius of the larger vessels in $\mu \mathrm{m}$.

The area densities of each class are computed for each section as a unit-less ratio between the amount of pixels belonging to that specific class and the total amount of pixels in the section. Fig. 2b shows the binary map of the capillaries in the five sections. We compute the vessel density (VD), the capillary density (CD) and the background density (BD) in the SRL. These abbreviations will be used from now on in this paper. In the DRL, we assume that only capillaries are present, so here we use the same segmentation model as in the SRL but combine the classes for capillaries and larger vessels. In the SRL, we compute the vessel radius of the larger vessels by first median filtering the binary class mask with a $3 \times 3$ neighborhood. We then compute the skeletonization of the output and compute the signed Euclidean distance field to the pixel center in order to obtain the distance to the closest boundary. In order to visualize the vessel thickness (Fig. 2c), the radius is plotted by dilating it with a disk shaped structure.

For every scan, the mentioned metrics in the five areas and in the two layers are concatenated into a feature vector, which is used in the analysis. The complete approach was implemented in MATLAB v9.5 (Mathworks, Inc.).

\section{$3 \quad$ Validation of approach}

The robustness of the proposed method is validated by applying it to data from healthy subjects acquired under three different scenarios but with an expectancy of no significant changes in the capillary network. We therefore hypothesize that there will be limited variation in the extracted features for each subject throughout the different scans. In order to test the feasibility of the method for abnormal eyes, we apply the method on OCTA data from cataract patients before and after surgery. We here hypothesize that there will be more variation in the extracted features. 


\subsection{Data and scanning protocol}

All data are acquired using either a swept source DRI OCT Triton or a swept source DRI OCT-1 Model Triton (plus), both from Topcon Medical Systems, Inc. The size of the scanning frame is $3 \times 3 \mathrm{~mm}^{2}$ centered on the macular region. The participants are instructed to fixate on a central object in order for the fovea to be located in the center. The retinal tissue layers are automatically segmented by the Topcon Advanced Boundary Algorithm (Topcon Medical Systems, Inc.). However, if the operator deems it necessary, the retinal layers are adjusted manually in IMAGEnet 6 , a browser-based software provided by Topcon Medical Systems, Inc. The layers are accessed in order to find the foveal center for the post-processing. Due to displacements from motion artefacts, different scans of the same patient are inspected in order to determine the center across scans. En face angiograms of the superior retinal layer (SRL) and the deep retinal layer (DRL) are exported in JPG format. The OCTA images have dimension $320 \times 320$ pixels with a pixel resolution of $9.38 \mu \mathrm{m}$. The OCTA images are included if the image quality provided by the Triton OCT is 50 or more, if there are no blinkor movement artefacts, and if an area with a radius of $1 \mathrm{~mm}$ from the foveal center is visible.

Healthy data set (scenarios H1, H2, and H3) To develop and validate the analysis method, we have chosen to acquire a high quality data set containing OCTA images from 10 healthy subjects at three different scenarios denoted H1, $\mathrm{H} 2$ and $\mathrm{H} 3$. In scenario H1, the pupil is undilated, in scenario $\mathbf{H 2}$, the pupil is medically dilated (using eye drops; Tropicamid (Mydriacyl) 1\%), and in scenario H3, the pupil is dilated and the subject has just walked briskly 5-7 flight of stairs. Between the first and second scenario, the optical quality of the eye changes because dilating the pupil allows more light to enter and exit the eye. We can hence test the effect of the amount of entered light. Between the second and the third scenario, we can test the robustness of the method with respect to potential perfusion changes from the exercise. The OCTA recordings are repeated until the quality-requirements described above are fulfilled. All subjects have normal visual acuity and no systemic or ocular disease. The visual acuity is measured in ETDRS letters. When converted into Snellen equivalent, the mean best corrected visual acuity is 1.25 (range 1.0 to 2.0 ). Maximal refraction is within $+/-3 \mathrm{D}$. We examine one eye from each of the 10 subjects, where 5 are male and 5 are female, and there are 5 left eyes and 5 right eyes. The mean age is 32.4 years with a standard deviation of 8.5.

Cataract data set (scenarios C1 and C2) For investigating how the method performs with lower quality data, OCTA images from cataract patients who underwent cataract surgery are obtained preoperatively (denoted C1) and three weeks postoperatively (denoted C2). The pupil is medically dilated using eye drops (Tropicamid (Mydriacyl) 1\% and Phenylephrine (Metaoxedrin) 10\%) on the preoperative images but not on the postoperative ones. OCTA images from 
44 consecutive patient records are reviewed by AMEE and JHE, and 10 of these are found to comply with the above mentioned quality criteria on both pre- and postoperative images. Of the 10 included patients, 8 are female and 2 are male, and there are 5 left eyes and 5 right eyes. The mean age is 70.3 years with a standard deviation of 5.5 .

\subsection{Statistical analysis}

In order to estimate the reproducibility of the method, we compare the different scanning scenarios of the healthy subjects. The intraclass correlation coefficient (ICC) is computed along with its $95 \%$ confidence intervals. The ICC describes both the correlation and the agreement of the measurements [17] and is used in most repeatability studies $[14,18,20]$. We use a single-rating, absoluteagreement and 2-way mixed-effect model using a MATLAB function provided by Salarian [19]. ICC values under 0.5 indicate poor reliability, between 0.5 to 0.75 moderate reliability, between 0.75 and 0.9 good reliability, and over 0.9 excellent reliability [15]. We use Bolt-Altman analysis to evaluate the agreement between the measurements, where we report the mean difference and the limits of agreement, which are set to 1.96 standard deviation, as done in [18]. We also perform a t-test for paired measurements as in $[1,20]$, where p-values less than 0.05 are considered significant.

We use factor analysis for analyzing the healthy data set and the cataract data set. Since we only have few observations and variables of high dimensions (the feature vector for every scan), we apply a principal axis factoring in order to decrease the number of dimensions. We use the varimax rotation to ease the interpretation of the factors. The sample means of each factor score are then compared using a multivariate two-sided analysis of variance (MANOVA) for the different groups as well as between all subjects. These statistical analyses are performed in SAS Studio, 3.8, SAS Institute Inc., Cary, NC, USA, and the reported p-values are the Wilks' lambda statistic.

\section{Results}

Fig. 3a-d show a good quality OCTA scan from a healthy subject and the resulting segmentation and radius information. Similarly, Fig. 3e-h show a reduced quality OCTA scan from a healthy subject included in the study. The average of the extracted metrics (used in the feature vector) of the healthy patients can be seen in Table 1 . The ICC and Bolt-Altman analysis for the healthy subjects and cataract patients are reported in Table 2.

We apply a factor analysis on the healthy subjects containing 30 observations where each has a 20-dimensional variable. Six factors are selected, which account for $84.65 \%$ of the variation in the data. An arbitrary threshold of \pm 0.5 is applied to ease the interpretation of the rotated factor pattern. The linear combinations of the resulting variables explaining each factor are shown in Table 3. When applying the MANOVA to the factor scores, we do not observe an effect 
Table 1. Mean and standard deviation of the features for all healthy subjects in all three scenarios (F: Fovea, I: Inferior, S: Superior, N: Nasal, T: Temporal, VD: Vessel density, CD: Capillary density, VR: Vessel radius in $\mu \mathrm{m})$.

\begin{tabular}{llllll}
\hline & SRL-F & SRL-I & SRL-S & SRL-N & SRL-T \\
\hline VD & $0.0025(0.0031)$ & $0.047(0.018)$ & $0.053(0.020)$ & $0.026(0.015)$ & $0.034(0.029)$ \\
CD & $0.26(0.067)$ & $0.44(0.069)$ & $0.47(0.048)$ & $0.45(0.054)$ & $0.45(0.069)$ \\
VR & $5.89(5.52)$ & $13.66(1.62)$ & $14.73(2.01)$ & $13.24(2.91)$ & $12.70(2.28)$ \\
\hline & DRL-F & DRL-I & DRL-S & DRL-N & DRL-T \\
\hline CD & $0.21(0.075)$ & $0.61(0.062)$ & $0.61(0.055)$ & $0.57(0.061)$ & $0.55(0.067)$
\end{tabular}

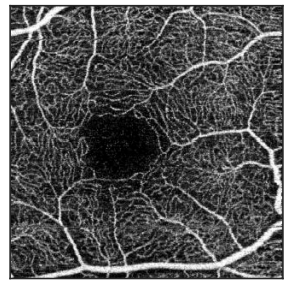

(a) SRL

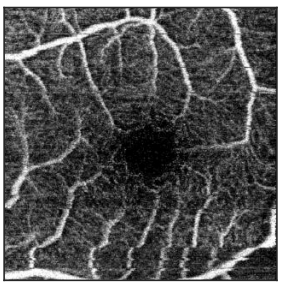

(e) SRL

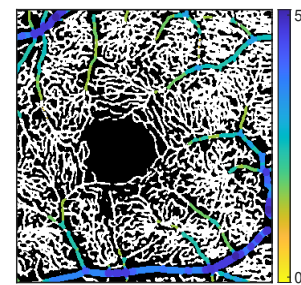

(b) SRL output

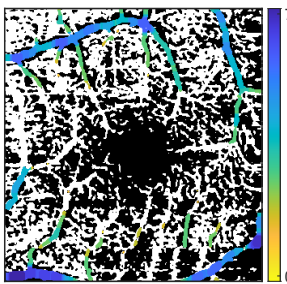

(f) SRL output

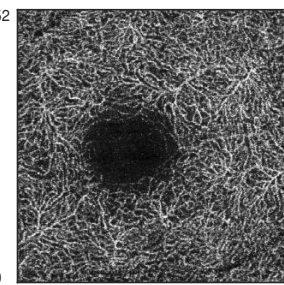

(c) DRL

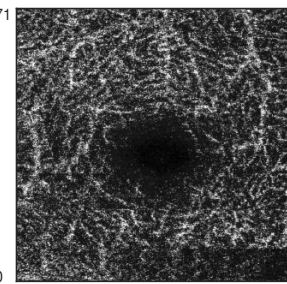

(g) DRL

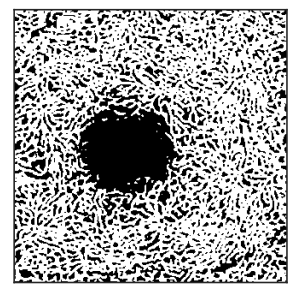

(d) DRL output

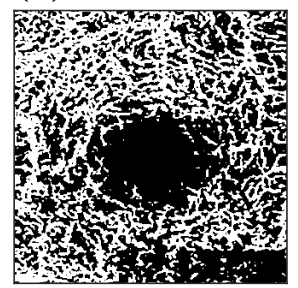

(h) DRL output

Fig. 3. Examples of segmentation output. Subfigures (a)-(d) illustrate the quantification of a good and clear OCTA, and (e)-(h) illustrate the quantification of a blurry OCTA. Subfigures (b) and (f) show the larger vessel radius in $\mu \mathrm{m}$.

in-between the three pupil scenarios $(\mathrm{p}=0.9513)$, but there is a highly significant difference between the different subjects $(\mathrm{p}<0.0001)$. We also perform a factor analysis of the cataract patients in a similar way. Here, we obtain different linear combinations describing 7 factors (accounting for $89.15 \%$ of the variation in the data). We do not observe a significant difference between the pre- and postoperative scans $(\mathrm{p}=0.2471)$, but the difference between the subjects is still very significant $(\mathrm{p}<0.0001)$.

\section{Discussion}

The relatively high Dice and Jaccard scores of the validation image in Fig. 1e indicate an adequate segmentation performance of the proposed dictionary model. A typical misclassification seen in our results is when bigger capillaries and 
Table 2. Comparisons between the different groups, H1: Healthy with undilated pupil, H2: Healthy with dilated pupil, H3: Healthy with dilated pupil after exercise, C1: Cataract patients preoperative, C2: Cataract patients postoperative. Reporting the MD: mean difference, LoA: limits of agreement, ICC: Intraclass correlation coefficient, CI: confidence interval. For the variables VD: Vessel density, CD: Capillary density, BD: Background density, VR: Vessel radius.

\begin{tabular}{ccccccc}
\hline \multicolumn{3}{c}{ Comparison } & \multicolumn{2}{c}{ Agreement } & Reliability & $\begin{array}{c}\text { Pairwise } \\
\text { comparison }\end{array}$ \\
\hline Group 1 Group 2 & Metric & MD & LoA & ICC (95\% CI) & P-value \\
\hline H1 & H2 & SRL CD & -0.0057 & $-0.11 / 0.10$ & $0.86(0.76,0.92)$ & 0.47 \\
H1 & H2 & SRL VD & -0.0014 & $-0.020 / 0.018$ & $0.93(0.88,0.96)$ & 0.32 \\
H1 & H2 & SRL BD & 0.0071 & $-0.11 / 0.12$ & $0.85(0.78,0.92)$ & 0.41 \\
H1 & H2 & SRL VR & -0.68 & $-6.32 / 4.97$ & $0.79(0.65,0.87)$ & 0.10 \\
H1 & H2 & DRL CD & -0.011 & $-0.14 / 0.12$ & $0.92(0.86,0.95)$ & 0.23 \\
\hline H2 & H3 & SRL CD & 0.0039 & $-0.10 / 0.11$ & $0.86(0.77,0.92)$ & 0.67 \\
H2 & H3 & SRL VD & -0.00050 & $-0.018 / 0.017$ & $0.94(0.89,0.96)$ & 0.69 \\
H2 & H3 & SRL BD & -0.0027 & $-0.11 / 0.11$ & $0.88(0.79,0.93)$ & 0.74 \\
H2 & H3 & SRL VR & -0.50 & $-4.36 / 3.36$ & $0.89(0.81,0.94)$ & 0.079 \\
H2 & H3 & DRL CD & 0.0040 & $-0.12 / 0.11$ & $0.93(0.89,0.96)$ & 0.63 \\
\hline C1 & C2 & SRL CD & 0.049 & $-0.12 / 0.22$ & $0.65(0.36,0.81)$ & $<\mathbf{0 . 0 0 1}$ \\
C1 & C2 & SRL VD & 0.0026 & $-0.029 / 0.035$ & $0.80(0.67,0.88)$ & 0.27 \\
C1 & C2 & SRL BD & -0.052 & $-0.23 / 0.13$ & $0.68(0.41,0.83)$ & $<\mathbf{0 . 0 0 1}$ \\
C1 & C2 & SRL VR & 0.65 & $-6.90 / 8.20$ & $0.73(0.57,0.84)$ & 0.13 \\
C1 & C2 & DRL CD & -0.018 & $-0.18 / 0.14$ & $0.90(0.82,0.94)$ & 0.24 \\
\hline
\end{tabular}

smaller vessels are not distinguished. However, this is to be expected, as it is also difficult to manually set the border between the two classes. The quality of the OCTA image in Fig. 3e-h makes it difficult to distinguish the capillaries manually, and the algorithm is challenged as well.

\subsection{Discussion of healthy subjects}

The retinal capillary density is generally reported to be between $30-60 \%$, and the great variation in different studies is due to different OCTA devices and methods [13]. Our capillary density estimates lie within this range (see Table 1) and are hence realistic, although cross-study comparisons should be done with care. The ICC values in Table 2 between $\mathrm{H} 1$ and $\mathrm{H} 2$ all vary between moderate to excellent, being highest for SRL VD and lowest for SRL VR. Similar trends can be seen when comparing $\mathrm{H} 2$ and H3, although here all ICC values are higher. We also see that the mean differences all lie close to zero, although the radius seems to have a small bias as well as a higher dispersion in the measurements. When looking at the pairwise comparison, no significant difference is observed between the different scanning scenarios. One reason why the vessel radius stands out in high dispersion could be due to a greater variation in the segmentation at the 
Table 3. Factors for healthy subjects, where SRL: Superficial retinal layer, DRL: Deep retinal layer, F: Fovea, I: Inferior, S: Superior, N: Nasal, T: Temporal, VD: Vessel density, CD: Capillary density, BD: Background density, VR: Vessel radius.

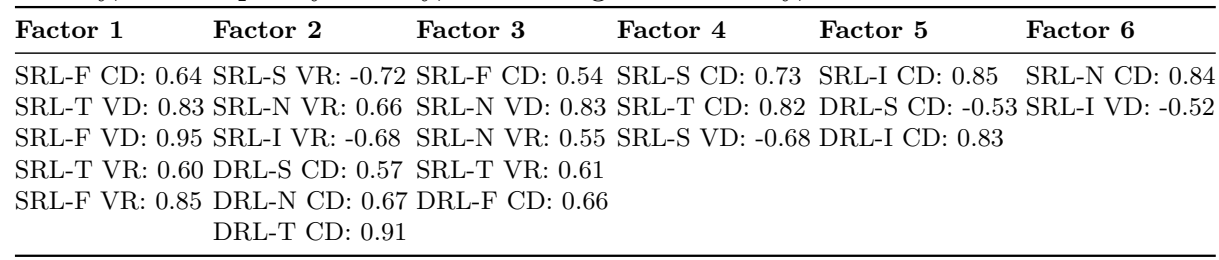

border between larger vessels and capillaries, and because of the hard margins between the ETDRS-sections.

When investigating the estimated factors from the factor analysis seen in Table 3, one can observe anatomical patterns. E.g. Factor 1 explains the capillary and vessel densities as well as the radius in the foveal and temporal region in the superficial layer. The MANOVA-test on the factor scores shows no difference between the three scenarios (H1, H2 and H3), and hence we can conclude that neither the dilation nor the exercise has an effect on the extracted metrics. We did however observe a difference between subjects, which means that each feature vector is representative of its subject, and hence that the extracted metrics are subject-specific.

\subsection{Discussion for cataract patients}

In Table 2, we see more variation in the quantitative metrics for the cataract patients, and decreased agreement and reliability compared to the healthy subjects. The quality of the cataract OCTA data is generally lower compared to the healthy subjects. Although it should be noted that all images used in this study were subjected to high quality requirements (compared to the common state of cataract patient data) in our selection process. The quality did however vary in both scenarios (pre- and postoperative). One reason for the great variation could be the optical deterioration of the lens preoperatively which results in a more blurry image, whereas the postoperative images were performed on an undilated pupil. Even though we did not observe a difference between undilated and dilated pupils for the healthy subjects, this could still have an effect for the older population. Obtaining an OCTA image with good image quality is challenging in cataract patients due to several reasons. The cataract itself worsens the optic quality of the image and patients may have difficulty focusing, which in turn may lead to small movements of the eye when the image is taken, causing artefacts. Furthermore, many cataract patients suffer from dry eyes and/or blepharitis, which causes irritation and blinking during the acquisition. Finally, the quality of the procedure is also dependent on the operator in terms of giving good instructions to the patient and securing a well focused image. 
Finer details, like capillaries, typically suffer most by the reduced quality in the cataract patients. This is supported by the significantly different SRL CD and SRL BD in the pairwise comparison. We did not observe any significant difference between the pre- and postoperative scans in the factor analysis, which can also be explained by the varying data quality in both scenarios - although a smaller $\mathrm{p}$-value $(\mathrm{p}=0.2471)$ suggests using a larger sample size.

Only a small circular area is used in the quantitative analysis. The method and the analysis could be improved by extending the area of interest through technical adjustments such that more information would be included. Ongoing improvements in OCTA systems to obtain a larger acquisition size would also facilitate this. Furthermore, additional metrics describing the microvasculature could be interesting to include. This can easily be added in the analysis pipeline, since the factor analysis is able to handle more variables than observations, while still explaining most of the variability in the data. This is only a preliminary investigation of the approach, and therefore there is still a need to evaluate it in other pathological conditions and on images captured in different devices.

\section{Conclusion}

This paper presents a new method that automatically quantifies the retinal vasculature in the superficial and deep layer. This is done through feature-based segmentation of the larger vessels and capillaries and through extracting metrics like densities and vessel radius from the segmentation. The method is validated on three repeated scans on healthy subjects in normal state (undilated pupil), with dilated pupil, and dilated pupil combined with exercise. The repeated scans are highly correlated showing that the reproducibility of our method is good. We do not see a difference between the scanning scenarios, although there is a difference between each subject demonstrating that the method is sensitive to variation in the structure of the vasculature. We also show that the method can be applied to cataract operated patients. This shows great promise for extending the method in order to investigate if there are features in the microvasculature that are important for the risk of developing macular edema after cataract surgery.

Acknowledgements We would like to thank Professor Emeritus Knut Conradsen, DTU Compute, and Assistant Professor Anders Nymark, DTU Compute, for valuable assistance and guidance in the statistical analysis.

\section{References}

1. Al-Sheikh, M., et al.: Repeatability of automated vessel density measurements using optical coherence tomography angiography. Br J Ophthalmol 101(4), 449452 (2017)

2. Campbell, J., et al.: Detailed vascular anatomy of the human retina by projectionresolved optical coherence tomography angiography. Sci Rep 7, 42201 (2017) 
3. Chu, Z., et al.: Quantitative assessment of the retinal microvasculature using optical coherence tomography angiography. J. Biomed. Opt 21(6), 066008 (2016)

4. Dahl, A., Larsen, R.: Learning dictionaries of discriminative image patches. In: Proc. BMVC. pp. 77.1-77.11. BMVA Press (2011)

5. Dahl, A.B., Dahl, V.A.: Dictionary snakes. In: Pattern Recognition (ICPR), 2014 22nd International Conference on. pp. 142-147. IEEE (2014)

6. Dahl, A.B., Dahl, V.A.: Dictionary based image segmentation. In: Scandinavian Conference on Image Analysis. pp. 26-37. Springer (2015)

7. Durbin, M.K., et al.: Quantification of retinal microvascular density in optical coherence tomographic angiography images in diabetic retinopathy. JAMA Ophthalmol. 135(4), 370-376 (2017)

8. Eladawi, N., et al.: Early diabetic retinopathy diagnosis based on local retinal blood vessel analysis in optical coherence tomography angiography (octa) images. Med. Phys. 45(10), 4582-4599 (2018)

9. Gadde, S.G., et al.: Quantification of vessel density in retinal optical coherence tomography angiography images using local fractal dimension. Invest Ophthalmol Vis Sci 57(1), 246-252 (2016)

10. Group, E.T.D.R.S.R.: Classification of diabetic retinopathy from fluorescein angiograms: Etdrs report number 11. Ophthalmology 98(5), 807-822 (1991)

11. Hwang, T.S., et al.: Automated quantification of capillary nonperfusion using optical coherence tomography angiography in diabetic retinopathy. JAMA Ophthalmol. 134(4), 367-373 (2016)

12. Iafe, N.A., et al.: Retinal capillary density and foveal avascular zone area are agedependent: quantitative analysis using optical coherence tomography angiography. Invest Ophthalmol Vis Sci 57(13), 5780-5787 (2016)

13. Kashani, A.H., et al.: Optical coherence tomography angiography: A comprehensive review of current methods and clinical applications. Prog Retin Eye Res. 60, 66-100 (2017)

14. Kim, A.Y., et al.: Quantifying microvascular density and morphology in diabetic retinopathy using spectral-domain optical coherence tomography angiography. Invest Ophthalmol Vis Sci 57(9), OCT362-OCT370 (2016)

15. Koo, T.K., Li, M.Y.: A guideline of selecting and reporting intraclass correlation coefficients for reliability research. J Chiropr Med 15(2), 155-163 (2016)

16. Mastropasqua, R., et al.: Foveal avascular zone area and parafoveal vessel density measurements in different stages of diabetic retinopathy by optical coherence tomography angiography. Int J Ophthalmol. 10(10), 1545 (2017)

17. McGraw, K.O., Wong, S.P.: Forming inferences about some intraclass correlation coefficients. Psychological methods 1(1), 30 (1996)

18. Rabiolo, A., et al.: Comparison of methods to quantify macular and peripapillary vessel density in optical coherence tomography angiography. PloS one 13(10), e0205773 (2018)

19. Salarian, A.: Intraclass Correlation Coefficient (ICC) Matlab Central File Exchange. https://se.mathworks.com/matlabcentral/fileexchange/22099-intraclasscorrelation-coefficient-icc (2016), retrieved 5 Feb 2019

20. Shoji, T., et al.: Reproducibility of macular vessel density calculations via imaging with two different swept-source optical coherence tomography angiography systems. Transl Vis Sci Techn 7(6), 31-31 (2018) 